\title{
Person-centered healthcare and integrated public health
}

\author{
*Corresponding Author: \\ Amy Price \\ Department of Continuing Education \\ University of Oxford Rewley House, 1 \\ Wellington Square \\ Oxford OX1 2JA (UK) \\ E-mail: dr.amyprice@gmail.com
}

Amy Price $^{a *}$, Rakesh Biswas ${ }^{b}$, Akshay Anand ${ }^{c}$

a Department of Continuing Education, University of Oxford, Oxford, UK

${ }^{b}$ Department of Medicine, IQ City Medical College, Durgapur, West Bengal, India

'Neuroscience Research Lab, Department of Neurology, PGIMER, Chandigarh, India

The public brings to the table valuable perspectives learned from living with the health problems research works to solve [1]. Patients as research partners in the clinic, at home, and in the lab can provide the fine details for a successful implementation that differentiate a research finding that sits expensive and unused from primary care that improves quality of life $[2,3]$. Life experiences can reveal practical wisdom for prioritizing the health questions that matter to patients [4]. It is possible that contributions by patient research partners may go unreported, and this can be compounded by role confusion where the terms "public involvement", "community engagement", "citizen support", and "quantified self-groups" are used interchangeably [5]. We know from a multiplicity of research that priorities recorded by researchers on behalf of patients may not be the same as those that are important to patients [6]

Uncertainty in chronic illness is a giant we all face. Currently, the traditional option is to wait patiently in crowded offices where time is at a premium and satisfactory queries to questions of survival and care are rare. The kind of questions patients and doctors both seek are very similar.

This can be captured by these concerns: "where is the problem located in my system?" (doctor's anatomical diagnosis), "why is this happening?" (etiological diagnosis), and specifically "why is the ailment happening to me or my loved one?", "what can be done now to right this condition?", and finally and tragically "why are the medicines not working?" (prognosis) and "what are my other options?".

Patients want to help and are willing to share their experiences and their data, but they need assurance their vulnerability will not be exposed to those who can harm them. Most would agree to data sharing with some caveats. People prefer personal data in transferable formats so they can control who to share it with and can download data to share across operating systems [7]. Patients would welcome the appearance of aggregated data, such as a dashboard where they can compare their lives and chosen interventions with the fates of populations before and concurrent to them. Patients want updates tailored to their needs. This requires the incubation of person-centered health with public health to increase information literacy.

In recent years, online consultations to address these queries have become a trend both among care-givers and care-seekers [8], and yet the current medical education curriculum (whose primary beneficiary is the patient) has not been able to create the kind of knowledge, attitude, and skills necessary for this evolving area [9]. Also one of the reasons why most people are unable to get answers to their queries is because of the paucity of solutions for many chronic, complex illnesses with multi-morbidity in spite of decades of advances in health research. It is possible that "multi-morbidity" is the manifestation of interconnected physiological network processes within an individual in their socio-cultural environment viz. genomic, metabolomic,proteomic, neuroendocrine, immune, and mitochondrial bioenergetic elements, as well as social, environmental, and healthcare networks. The future of "multi-morbidity" management might become much more discerning by combining the balancing of physiological dysregulation with targeted personalized biotechnology interventions. These may include small-molecule therapeutics targeting specific cellular components of the stress response. Simultaneous community-embedded interventions that involve addressing psycho-socio-cultural impediments would help to strengthen personal/social resilience and enhance social capital [10].

The focus area includes "patient-centered outcomes research," which is an emergent research method evolving since 2010 [11]. This area helps to meet the needs of the patient's requirements and can provide alignment with their personal values. Mobilization and training of human resources is needed to answer those requirements with effectual, practical, and innovative solutions. This fosters social outreach not only to the patients and the individuals seeking preventive therapeutics/ 


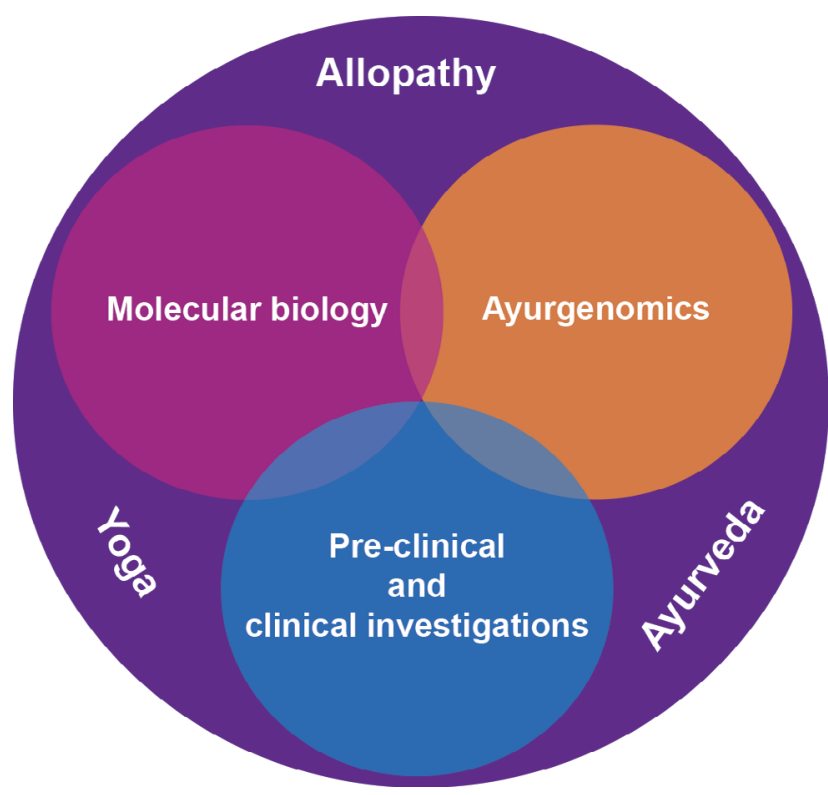

Rationale of a proposed incubation center.

practices, but also reaches to the students at primary level so that their minds are ignited at an early stage about the existence of age-old practices, current therapeutic measures, and the history of their evolution as well as the need for innovation.

We aspire to a future where healthcare transformation is developed through shared learning between health professionals, scientists, students/scholars, citizens, and patients with chronic complex illnesses.

We propose to elaborate and objectify these ideals through the construction of incubation centers that may include these initiatives:

1. To involve out-patient departments (OPDs) from leading health institutes and hospitals for the recruitment of complex patients with multi-morbidity in whom modern medical treatment has failed into an incubation center for evidence-informed innovative strategies toward further monitoring and treatment. This recruitment of patients will also involve organizing mega health literacy campaigns free to patients to create awareness about health, help, and available evidence for early detection and treatment. This could be managed with the help of volunteer healthcare workers, technicians, and allied paramedics, exploiting the social skills of non-government organizations (NGOs).

2. To assemble various healthcare systems under one roof (allopathy, Ayurveda, Yoga, genetics, stem cells, and research) for wellness and holistic treatments including teams with respective expertise. One rationale for this is that cultural traditions can be twisted by for-profit junk scientists. The harms caused by this exposure to bad health science can reduce treatment efficacy and endanger patients. In an incubation center, patients can be treated holistically and with full transfer of information between practitioners of each discipline. In this way, case studies, between-person interventions, and even clinical trials can explore the evidence base for complementary and emergent care.

3. In accordance with patient preferences and long-held Indian traditions, it was suggested patients could be stratified for a regime facilitating categorization through the doshic and prakriti (Vata, Pitta, and Kapha as outlined by Ayurveda) variations and further implementing these dimensions in personalized medicine. This could form a specialized branch of Ayurgenomics.

4. To install affordable, accessible genetic tools for the early detection, counseling, best intervention, and prevention of avoidable disease in vulnerable families.

5. To implement pre-clinical investigations and establish the efficacy of the integrative medications by in vitro, in vivo, and patient-driven research. The study would involve the comparative study between herbals, Yoga, and biologicals and could contribute to citizen science research involvement.

6. Better awareness and management of individual health and illnesses through a networked system approach to include awareness about lifestyle and non-communicable diseases and as a center for community-based research and understanding uncertainty and risk. It also involves awareness-based interactive learning for schools and college students as part of the social outreach program of the center.

7. The development of a working model of a "participatory learning ecosystem for practising evidence-informed multidisciplinary integrative medicine" where instead of just a single doctor, the patient gets the benefit of a global (and local) network of health professionals and scientists (including citizen scientists) who optimize the patient's information requirements in a manner that can influence their chronic disease problem in the direction of positive outcomes. This model facilitates a culturally sensitive, literacy- considerate incubator for testing multi-criterion, analytic, informed, shared decision making that incorporates simple sliders where patients can indicate values and preferences against preset evidence-based algorithms.

8. Employment generation: a large workforce will be "task-shifting" traditional roles of "individual patient information communication management". This was till date a preserve of physicians, but it is no longer manageable by them due to the sheer volume of information growth in medicine.

9. This system hopes to rationalize healthcare budgets through such measures and by the integration of health learning camps. This will provide a vehicle for shared recom mendations from interdisciplinary panels of experts for the implementation of efficacy and comparative effectiveness rather than the constraints and inconsistencies of consensus by opinion.

10. In devising a suitable MD-PhD and PhD-MD program in integrative medicine that can showcase the fruits of the innovative solutions proposed and developed during the entire project duration. 
11. In bringing together the platforms of data management, patient-friendly open notes for the clinical record, and publications through integrative medicine and neuroscience communication.

12. In teaching the philosophy and Sanskrit for the elucidation and decoding of age-old practices contained in the Indian texts for MD-PhD and PhD-MD students for the better understanding of Indian forms of medicine and integration within the international healthcare system.

\section{Acknowledgements}

We are thankful for the role of the organizations Tabula Rasa and Empower 2 Go, whose citizens, physicians, students, and social workers aided in defining the needs of medical citizens and discussed the best ways to reach them as shared decision makers and collaborators in this age of personal medicine and advanced technology. They guided us on including the needs and expanding roles of the vulnerable but active patient in the digital age.

\section{Disclosure statement}

To the best of our knowledge, no conflicts of interest exist. Reprinted with permission from Integrative Medicine International.

\section{References}

1. Boote J, Wong R, Booth A: “Talking the talk or walking the walk?" A bibliometric review of the literature on public involvement in health research published between 1995 and 2009. Heal Expect 2015;18:44-57.
2. Price A, Biswas T, Biswas R: Person-centered healthcare in the information age: experiences from a user driven healthcare network. Eur J Pers Cent Healthc 2013;1:385.

3. Richards T, Snow R, Schroter S: Co-creating health: more than a dream. BMJ 2016;4550:i4550.

4. Crocker JC, Boylan A-M, Bostock J, Locock L: Is it worth it? Patient and public views on the impact of their involvement in health research and its assessment: a UK-based qualitative interview study. Heal Expect 2016, DOI: 10.1111/hex.12479.

5. Brett J, Staniszewska S, Mockford C, Herron-Marx S, Hughes J, Tysall $\mathrm{C}$, et al: Mapping the impact of patient and public involvement on health and social care research: a systematic review. Heal Expect 2014;17:637-650.

6. Snow R, Crocker JC, Crowe S: Missed opportunities for impact in patient and carer involvement: a mixed methods case study of research priority setting. Res Involv Engagem 2015;1:7.

7. Price A, Liew SM, Kirkpatrick J, Price J, Lopreto T, Nelken Y: Mind the gap in clinical trials: a participatory action analysis with citizen collaborators. J Eval Clin Pract 2017;23:178-184.

8. Purkayastha S, Price A, Biswas R, Jai Ganesh AU, Otero P: From dyadic ties to information infrastructures: care-coordination between patients, providers, students and researchers. Yearb Med Inform 2015;10: 68-74.

9. Bera K, Seth B, Biswas R: Conversational learning among medical students: harnessing the power of web 2.0 through user driven healthcare. Ann Neurosci 2013;20:37-38.

10. Sturmberg JP: Multimorbidity and chronic disease: an emergent perspective. J Eval Clin Pract 2014;20:508- 512.

11. Gabriel SE, Normand S-LT: Getting the methods right - the foundation of patient-centered outcomes research. N Engl J Med 2012;367: 787-790.

doi: 10.38205/imcr.010103 